\title{
VI3. EXPRESSION OF PRO INFLAMMATORY GENES AFTER YELLOW FEVER VACCINATION IN HUMANS: IMPLICATIONS FOR ACQUIRED IMMUNE RESPONSE.
}

Tamiris Azamor da Costa Barros ${ }^{1}$; Andréa Marques Vieira da Silva ${ }^{1}$; Caroline Xavier Carvalho²; Lucia E. Alvarado-Arnez ${ }^{2}$; Camilla Bayma Fernandes; Denise Cristina de Souza Matos ${ }^{1}$; Milton Ozório Moraes²; Patrícia Cristina da Costa Neves ${ }^{1}$.

${ }^{1}$ Bio- Manguinhos/ Fiocruz;

${ }^{2}$ Instituto Oswaldo Cruz/ Fiocruz.

INTRODUCTION The vaccine against Yellow Fever is one of the most effective ever made with more than 540 million doses administered globally. It confers long term protection after a single dose. Vaccination with YF17D virus induces polyvalent immune responses, with a mixed Th1/Th2 CD4+ cell profile, which results in robust TCD8+ responses and high titers of neutralizing antibody. Despite these characteristics, very little is known about the cellular and molecular mechanisms that lead to such good immune response. Moreover, although rarely observed, some serious adverse events may occur after vaccination and the mechanisms for its development are not yet understood. It is now clear that early events following YF17D immunization have a key role in determining the strength and quality of the adaptive immune response. In this manner, our group has previously shown that primary immunization of humans, monkeys and mice, with YF vaccine resulted in: i) early synthesis of IFN- $\gamma$ and ii) correlation between the magnitude of early IFN- $\gamma$ release and the quality of acquired immune responses in mice. Interestingly, another group has already shown that early IFN- $\gamma$ and IL-6 responses after YF immunization are correlated with the levels of neutralizing antibodies in humans.

OBJECTIVE Herein we have investigated early IFNs and IL-6-related gene expression in volunteers immunized with YF17DD.

METHODOLOGY Blood samples from fifteen volunteers immunized with 17DD were collected $0,4,7,10$ and 30 days after immunization. The peripheral blood mononuclear cells were isolated and submitted to RNA extraction, followed by cDNA synthesis. The q-PCR assay was carried out in samples from 0, 4, 7 and 10 days p.i, 
to quantify expression of CLEC5A, IFNG, IL6, RNAseL, IRF7, IRF9, STAT2 and OAS1. Antibodies against yellow fever were quantified using Virus Binding inhibition assay.

RESULTS It was possible to observe an increase in the expression of all genes studied, mainly OAS1 and IRF7. All of them, except IFNG and IL6, were significantly correlated with the development of antibodies against Yellow Fever Virus. In addition, we show a differential expression of CLEC5A gene as early as four days after immunization, to our knowledge this has not been previously reported. This up or down regulation in gene expression was also correlated with the levels of yellow fever antibodies, indicating a role for this lectin receptor expressed in cells from the myeloid lineage during immunological response development after YF17DD inoculation. It is noteworthy that CLEC5A has also been implicated in several mechanisms during Dengue and Japanese Encephalitis infections, two flaviviruses related to YF vírus.

CONCLUSION These results indicated that early events after yellow fever immunization are, in fact, related to antibodies development. Moreover, we described a new gene, CLEC5A, which may be involved in yellow fever response.

KEYWORDS Yellow Fever vaccine, early gene expression, acquired response. 